\title{
Singlet portal to the hidden sector
}

\author{
Clifford Cheung ${ }^{a, b}$ and Yasunori Nomura ${ }^{a, b, c}$ \\ ${ }^{a}$ Berkeley Center for Theoretical Physics, University of California, \\ Berkeley, CA 94720, U.S.A. \\ ${ }^{b}$ Theoretical Physics Group, Lawrence Berkeley National Laboratory, \\ Berkeley, CA 94720, U.S.A. \\ ${ }^{c}$ Institute for the Physics and Mathematics of the Universe, University of Tokyo, \\ Kashiwa 27\%-8568, Japan \\ E-mail: clifford.cheung@berkeley.edu, ynomura@lbl.gov
}

ABSTRACT: Ultraviolet physics typically induces a kinetic mixing between gauge singlets which is marginal and hence non-decoupling in the infrared. In singlet extensions of the minimal supersymmetric standard model, e.g. the next-to-minimal supersymmetric standard model, this furnishes a well motivated and distinctive portal connecting the visible sector to any hidden sector which contains a singlet chiral superfield. In the presence of singlet kinetic mixing, the hidden sector acquires a light mass scale in the range $0.1-100 \mathrm{GeV}$ induced by electroweak symmetry breaking. In theories with $R$-parity conservation, superparticles produced at the LHC cascade decay into hidden sector particles. Since the hidden sector singlet couples to the visible sector via the Higgs sector, these cascades produce a Higgs boson in an order $0.01-1$ fraction of events. Furthermore, supersymmetric cascades typically produce highly boosted, low-mass hidden sector singlets decaying visibly, albeit with displacement, into the heaviest standard model particles which are kinematically accessible. We study experimental constraints on this broad class of theories, as well as the role of singlet kinetic mixing in direct detection of hidden sector dark matter. We also present related theories in which a hidden sector singlet interacts with the visible sector through kinetic mixing with right-handed neutrinos.

KeYwords: Supersymmetric Effective Theories, Supersymmetry Breaking

ARXIV EPRINT: 1008.5153 


\section{Contents}

1 Introduction $\quad 1$

2 Basic setup 3

3 Spontaneous scale generation $\quad 5$

$\begin{array}{lll}4 & \text { The portal } & 6\end{array}$

5 To the hidden sector $\quad 8$

$\begin{array}{lll}6 & \text { From the hidden sector } & 10\end{array}$

$\begin{array}{lll}7 & \text { Constraints } & 14\end{array}$

8 Dark matter $\quad 15$

$\begin{array}{lll}9 & \text { Discussion and conclusions } & 17\end{array}$

\section{Introduction}

Physics beyond the standard model has been largely devoted to an emerging understanding of the fundamental constituents of matter at ever higher energies. In more recent years, however, some of the focus has shifted away from this "vertical" line of thinking towards a more "horizontal" perspective concerned with the possibility of hidden sectors which are weakly coupled to the visible sector but at the same time comprised of particles at observable mass scales. Indeed, the existence of multiple separate sectors is quite plausible in the context of string theory, which often predicts a number of geographically sequestered sectors $[1-6]$.

Theories with such light hidden sectors are particularly well motivated and exhibit rich phenomenology if there is weak scale supersymmetry. With supersymmetry, mass scales of these sectors can be naturally at or below the weak scale, since they can be dominantly generated by supersymmetry breaking effects induced by interactions with the visible sector or "mandatory" gravity mediation. Moreover, supersymmetry can offer a unique window into hidden sectors via decay of the lightest observable-sector supersymmetric particle (LOSP). As such, phenomenology depends crucially on specific operators connecting visible and hidden sector particles.

In general, there may exist heavy mediator particles of mass $M_{*}$ which serve as a bridge between the visible and hidden sectors. At low energies, this typically implies that the two sectors couple only through higher dimension operators suppressed by $M_{*}$. There 
are, however, two exceptions to this expectation. First, if the hidden sector contains a U(1) gauge field, loops involving heavy mediators can generate a marginal operator [7]

$$
\mathcal{L}=\chi \int d^{2} \theta \mathcal{W}^{\alpha} \mathcal{W}_{\alpha}^{\prime}+\text { h.c. }
$$

where $\mathcal{W}^{\alpha}$ and $\mathcal{W}_{\alpha}^{\prime}$ are $\mathrm{U}(1)$ hypercharge and hidden sector field-strength superfields. This scenario has been extensively studied in literature, for example in [8-27]. In this paper we discuss an alternative possibility: if both the visible and hidden sectors contain singlet chiral superfields, $S$ and $S^{\prime}$, then a marginal kinetic mixing operator

$$
\mathcal{L}=\epsilon \int d^{4} \theta S^{\dagger} S^{\prime}+\text { h.c. }
$$

can persist at low energies, no matter the scale of new physics, $M_{*}$. The size of the coefficient $\epsilon$ is typically a one-loop factor or less, $O\left(\lesssim 1 / 16 \pi^{2}\right)$. Note that any hidden sector which interacts with the visible sector via a marginal operator will essentially dictate the phenomenology - the effect of additional sectors interacting only through higher dimension operators will be subdominant.

We assume that the visible sector singlet interacts with fields in the minimal supersymmetric standard model (MSSM) through a superpotential term

$$
W=\lambda S H_{u} H_{d}
$$

where $H_{u}$ and $H_{d}$ are the up-type and down-type Higgs doublets, and $\lambda$ is an $O(\lesssim 1)$ coupling. Indeed, assuming the existence of an $R$-parity under which $S$ is even, this is the only renormalizable operator which can be written. ${ }^{1}$ Our analysis will be largely independent of any additional interactions involving $S$ - a special case is the usual nextto-minimal supersymmetric standard model (NMSSM), $\Delta W=\kappa S^{3} / 3$.

The framework defined by eqs. (1.2) and (1.3) leads to rich and distinctive phenomenology. Three main features are

- Spontaneous Scale Generation. After electroweak symmetry breaking, singlet kinetic mixing induces an effective linear term for $S^{\prime}$ in the superpotential, set by the scale $\Lambda_{\text {eff }}^{2}=\epsilon(\lambda / 2) v^{2} \sin 2 \beta+\cdots \approx O(0.1-100 \mathrm{GeV})$, where $v \equiv \sqrt{\left\langle H_{u}\right\rangle^{2}+\left\langle H_{d}\right\rangle^{2}}$ and $\tan \beta \equiv\left\langle H_{u}\right\rangle /\left\langle H_{d}\right\rangle$. As a result, hidden sector fields generically acquire vacuum expectation values (VEVs) of order $\Lambda_{\text {eff }}$, yielding light degrees of freedom at this scale. (If the contribution from gravity mediation is larger, the characteristic mass scale of the hidden sector may be set by that.)

- Higgs Production with Supersymmetry. Since the hidden sector typically contains an $R$-parity odd state which is lighter than the LOSP, a superparticle invariably cascades into states in the hidden sector. Because the hidden sector communicates with the visible sector only through $S$, which interacts with MSSM states only via the Higgs fields, these cascades necessarily produce the Higgs boson in an $O\left(10^{-2}-1\right)$ fraction of events, depending on $\Lambda_{\text {eff }}$ and the LOSP species. This leads to a minimum rate for high transverse-momentum Higgs production associated with significant missing energy.

\footnotetext{
${ }^{1}$ The case where $S$ is $R$-parity odd will be discussed in the final section.
} 
- Hidden Sector Cascades Return. Hidden sector singlets produced by supersymmetric cascades may decay back to standard model states, if they are even under $R$-parity. Since this process occurs through off-shell Higgs fields, the decay product is typically the heaviest possible state which is kinematically accessible. The decay rate scales roughly as $\Gamma \propto \epsilon^{2} y^{2}\left(m^{\prime} / v\right)^{2} m^{\prime}$, where $y$ is the Yukawa coupling of the final state and $m^{\prime}$ the mass of the hidden sector singlet. Because of the suppression due to $\epsilon^{2}, y^{2}$, and $\left(m^{\prime} / v\right)^{2}$, the vertex is generically displaced. The decay, however, may still occur inside the detector for natural values of $\epsilon$, so the decay products may be observed at colliders.

These features allow for distinguishing theories with singlet portal from alternative scenarios such as $\mathrm{U}(1)$ gauge kinetic mixing.

The organization of this paper is as follows. In section 2, we describe our basic setup. We study spontaneous scale generation in section 3, and interactions between the visible and hidden sectors in section 4 . In sections 5 and 6 , we describe physics of the "portal in" and the "portal out," i.e. processes converting visible sector states into hidden sector ones and vice versa. We discuss experimental constraints in section 7 , and study possible implications of this framework on dark matter in section 8. Finally, we conclude in section 9 , and present related theories of singlet kinetic mixing in which the hidden sector interacts with the visible sector through right-handed neutrinos.

\section{Basic setup}

Let us consider a scenario in which there exist two "separate" sectors, for example those geographically sequestered from each other along an extra dimension. These two sectors may still be connected through physics at some high energy $M_{*}$, e.g. at the compactification scale. This typically leads to a low energy effective theory in which the two sectors interact only through higher dimension operators suppressed by $M_{*}$.

However, if both sectors contain a singlet chiral superfield, $S$ and $S^{\prime}$, then the low energy theory may in general contain the marginal kinetic mixing operator in eq. (1.2). For example, this operator can be generated by loops of a heavy field $\Phi / \bar{\Phi}$ that interacts with $S$ and $S^{\prime}$ through the superpotential

$$
W=y S \Phi \bar{\Phi}+y^{\prime} S^{\prime} \Phi \bar{\Phi} .
$$

This yields a kinetic mixing operator with the coefficient

$$
\epsilon \approx \frac{y y^{\prime}}{16 \pi^{2}} \ln \frac{M_{*}}{M_{\Phi}}
$$

where $M_{\Phi}$ and $M_{*}$ are the mass of $\Phi / \bar{\Phi}$ and the ultraviolet cutoff, respectively.

In general, the precise structure of the heavy-field sector and its couplings to $S$ and $S^{\prime}$ are unknown, so the size of $\epsilon$ is model dependent. It is, however, reasonable to expect that $\epsilon$ is of order a one-loop factor or less, and in this paper we mainly focus on the range

$$
10^{-5} \lesssim \epsilon \lesssim 10^{-1}
$$




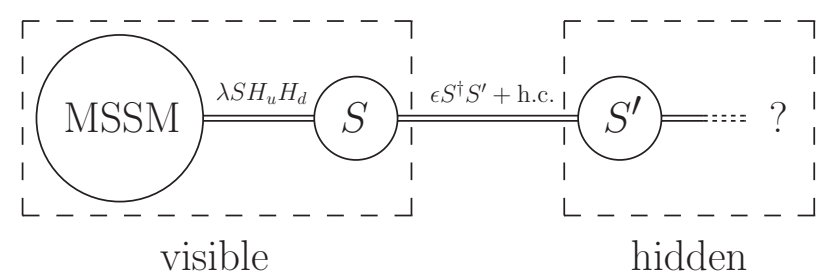

Figure 1. A schematic depiction of our setup. Integrating out heavy states typically induces a marginal kinetic mixing operator between singlet chiral superfields $S$ and $S^{\prime}$. The singlet $S$ couples with sizable strength to the MSSM, e.g. like in the NMSSM.

Since renormalization group evolution from $M_{\Phi}$ to the weak scale does not have a significant effect on the size of $\epsilon$, we consider the operator in eq. (1.2) with eq. (2.3) evaluated at the weak scale.

As described in the introduction, we assume that $S$ interacts with the MSSM states through the interaction in eq. (1.3). We therefore consider the following superpotential for the visible sector:

$$
W=\lambda S H_{u} H_{d}+\mu_{0} H_{u} H_{d}+f(S)
$$

where $f(S)$ is a holomorphic function of $S$. The conventional NMSSM corresponds to $\mu_{0}=0$ and $f(S)=\kappa S^{3} / 3$; but in general $\mu_{0}$ may be of order the weak scale, and $f(S)$ may contain terms linear or quadratic in $S$ with weak scale coefficients. We assume that $S$ and $H_{u, d}$ obtain nonvanishing VEVs after supersymmetry breaking, which we denote by

$$
x \equiv\langle S\rangle, \quad v_{u} \equiv\left\langle H_{u}\right\rangle, \quad v_{d} \equiv\left\langle H_{d}\right\rangle
$$

The supersymmetric mass term for $H_{u, d}$ (the $\mu$ term) is then given by $\mu=\mu_{0}+\lambda x$. A schematic depiction of the setup described here is given in figure 1.

Note that $S$ and $S^{\prime}$ may not be elementary singlets - the relevant renormalizable operators in eqs. (1.2) and (1.3) may exist if there are singlets $S$ and $S^{\prime}$ at the weak scale. However, if the compositeness scales for $S$ and $S^{\prime}$ are hierarchically smaller than the cutoff scale, then the size of $\epsilon$, as well as other interactions of $S$ and $S^{\prime}$, are suppressed accordingly. Below, we assume that this suppression is absent, either because $S$ and $S^{\prime}$ are elementary or because their compositeness scales are sufficiently high.

Finally, let us comment on the possibility that the gauge hierarchy might be destabilized in the presence of the visible sector singlet $S$. First, the problem may be avoided if the scale of fundamental supersymmetry breaking is sufficiently low, such as in low scale gauge mediation. On the other hand, if the scale of supersymmetry breaking is high, dangerous Kähler potential operators will generically be induced, yielding an unacceptably large tadpole for $S$ [28-30]. This problem can be solved if the theory possesses a (softly broken) discrete symmetry, or if anthropic selection plays a role in determining the weak scale. Because hierarchy destabilization is a generic pitfall of singlet extensions of the MSSM, we will not address it further in this paper. 


\section{Spontaneous scale generation}

The operator in eq. (1.2) spontaneously generates a scale of $O(0.1-100 \mathrm{GeV})$ in the hidden sector. To see this, let us denote the component fields of $S$ and $S^{\prime}$ by

$$
\begin{aligned}
S & =s+\sqrt{2} \theta \tilde{s}+\theta^{2} F_{S}, \\
S^{\prime} & =s^{\prime}+\sqrt{2} \theta \tilde{s}^{\prime}+\theta^{2} F_{S^{\prime}},
\end{aligned}
$$

and expand eq. (1.2) as

$$
\mathcal{L}_{\text {kin }}=\epsilon\left(-\partial^{\mu} s^{\dagger} \partial_{\mu} s^{\prime}+i \tilde{s}^{\dagger} \bar{\sigma}^{\mu} \partial_{\mu} \tilde{s}^{\prime}+F_{S}^{\dagger} F_{S^{\prime}}\right)+\text { h.c. }
$$

After electroweak symmetry breaking, $F_{S}^{\dagger}$ acquires a VEV which induces a tadpole for $F_{S^{\prime}}$. This term is equivalent to adding an effective Polonyi term to the hidden sector superpotential,

$$
W_{\mathrm{eff}}=-\Lambda_{\mathrm{eff}}^{2} S^{\prime}
$$

where

$$
\begin{aligned}
\Lambda_{\mathrm{eff}}^{2} & \equiv-\epsilon\left\langle F_{S}^{\dagger}\right\rangle=\epsilon\left(\frac{\lambda v^{2} \sin 2 \beta}{2}+\frac{d f(x)}{d x}\right) \\
& \approx O(0.1-100 \mathrm{GeV})^{2},
\end{aligned}
$$

corresponding to the range of $\epsilon$ quoted in eq. (2.3) and $1 \lesssim \tan \beta \lesssim 50$. Here the function $f$ was defined in eq. (2.4) and we have made the reasonable assumption that $d f / d x$ is not much greater than the weak scale.

Note that if the $d f / d x$ term is a subdominant contribution to $\Lambda_{\text {eff }}^{2}$, then $\Lambda_{\text {eff }}^{2} \propto \sin 2 \beta$ and may be significantly suppressed at large $\tan \beta$. This is an important difference from the case of gauge kinetic mixing of hypercharge and a hidden sector $\mathrm{U}(1)^{\prime}$. There electroweak symmetry breaking induces an effective Fayet-Iliopoulos term for the $\mathrm{U}(1)^{\prime}$ gauge field which goes as $\xi \propto \cos 2 \beta$ and is thus largely insensitive to $\tan \beta$ unless $\tan \beta \approx 1$ [27].

The effective Polonyi term in eq. (3.5) injects the scale $\Lambda_{\text {eff }}$ into the hidden sector. As a result, the masses of hidden sector fields are typically of this order. For instance, consider a simple hidden sector theory in which $S^{\prime}$ has a trilinear superpotential interaction. Together with eq. (3.4), the effective hidden sector superpotential is then

$$
W_{\text {hid }}=-\Lambda_{\text {eff }}^{2} S^{\prime}+\frac{\kappa^{\prime}}{3} S^{\prime 3} \text {. }
$$

The scalar potential is

$$
V_{\text {hid }}=\left|\kappa^{\prime} s^{\prime 2}-\Lambda_{\text {eff }}^{2}\right|^{2} .
$$

Thus, $x^{\prime} \equiv\left\langle S^{\prime}\right\rangle=\sqrt{\Lambda_{\text {eff }}^{2} / \kappa^{\prime}}$, and the vacuum aligns to preserve supersymmetry. ${ }^{2}$ Furthermore, $s^{\prime}$ and $\tilde{s}^{\prime}$ both acquire a mass $m^{\prime 2}=4\left|\kappa^{\prime} \Lambda_{\text {eff }}^{2}\right|$. For $O(\lesssim 1)$ values of $\kappa^{\prime}$, this implies a hidden sector singlet mass in the range

$$
m^{\prime} \approx O(10 \mathrm{MeV}-100 \mathrm{GeV}) .
$$

\footnotetext{
${ }^{2}$ Despite the presence of an effective Polonyi term, the vacuum typically adjusts to preserve supersymmetry. A notable exception is O'Raifeartaigh-like constructions, such as the one defined by $W_{\text {hid }}=-\Lambda_{\text {eff }}^{2} S^{\prime}+\mu^{\prime} T^{\prime} U^{\prime}+\lambda^{\prime} S^{\prime} T^{\prime 2}$.
} 
The spontaneous scale generation exhibited by this simple model is a generic feature of models with kinetically mixed singlets.

Of course, $m^{\prime}$ can exceed $\Lambda_{\text {eff }}$ if the hidden sector has additional sources of mass generation. In particular, this may occur if $W_{\text {hid }}$ contains explicit mass terms or if the hidden sector receives large supersymmetry breaking contributions, for example from gravity mediation. These effects are highly model dependent - explicit mass terms are easily forbidden by any number of chiral, $R$, or discrete symmetries, and the scale of supersymmetry breaking may be low, in which case gravity mediated contributions will be subdominant. Nevertheless, as to be as model independent as possible, the remainder of our discussion will be agnostic about the origin of $m^{\prime}$, and will consider the possibility that $m^{\prime}$ may be as large as the weak scale, regardless of the value of $\Lambda_{\text {eff }}$.

\section{The portal}

We now discuss the effective interactions between visible and hidden sector fields. To simplify the discussion, we consider only a single field $S^{\prime}$ in the hidden sector - the existence of possible additional fields will not affect our basic conclusions. The most general hidden sector superpotential is then written as

$$
W_{\text {hid }}=\frac{m^{\prime}}{2} S^{\prime 2}+\frac{\kappa^{\prime}}{3} S^{\prime 3}
$$

because we can always define the origin of $S^{\prime}$ so that the linear term in the superpotential vanishes (unless $\partial^{2} W_{\text {hid }} / \partial S^{\prime 2}=0$ in the original basis). Note that $W_{\text {hid }}$ includes the effect of the Polonyi term in eq. (3.4), as in eq. (3.6). For the model of eq. (3.6), for example, eq. (4.1) is obtained after the shift $S^{\prime} \rightarrow S^{\prime}+x^{\prime}$, so that $m^{\prime}=2 \sqrt{\kappa^{\prime}} \Lambda_{\text {eff }}$.

In what follows, we will assume $m^{\prime}, \kappa^{\prime} \neq 0$, which indeed represents the situation for generic hidden sectors. We will mostly ignore supersymmetry breaking effects in the hidden sector, which is typically a good approximation. (It is indeed a very good approximation if the dominant superparticle masses arise from gauge mediation in the visible sector.) The case where the supersymmetry breaking effects are important will be discussed briefly.

In general, interactions between the visible and hidden sector fields can be obtained by canonically normalizing fields, starting from the original Lagrangian containing kinetic mixing terms of eq. (3.3). For small $\epsilon$, however, there is a simple way to obtain the leading interactions between the two sectors, which we will present below.

Let us first consider the hidden sector fermion, $\tilde{s}^{\prime}$. At the leading order in $\epsilon$, the kinetic mixing between $\tilde{s}$ and $\tilde{s}^{\prime}$ in $\mathcal{L}_{\text {kin }}$ can be removed by the shift

$$
\tilde{s}^{\prime} \rightarrow \tilde{s}^{\prime}-\epsilon \tilde{s}
$$

This induces interactions between visible and hidden sector fields, $-\epsilon \tilde{s}\left(\partial / \partial \tilde{s}^{\prime}\right) \mathcal{L}_{\text {hid }}$, where $\mathcal{L}_{\text {hid }}$ denotes the hidden sector interaction Lagrangian. For the theory defined in eq. (4.1), the resulting term is $2 \epsilon \kappa^{\prime} s^{\prime} \tilde{s}^{\prime} \tilde{s}$. Note that an interaction term generated in this way always contains only a single visible sector field. 
Another important effect of the shift in eq. (4.2) is to induce a mass mixing, $\epsilon m^{\prime} \tilde{s}^{\prime} \tilde{s}$. As a result, the fermion mass matrix takes the following schematic form

$$
\mathcal{M}_{\text {fermion }}=\left(\begin{array}{c|c|c}
m \approx \text { weak scale } & \\
& \epsilon m^{\prime} \\
\hline \epsilon m^{\prime} & m^{\prime}
\end{array}\right),
$$

where the upper-left block corresponds to the neutralino mass matrix of the visible sector in the $\left\{\tilde{b}, \tilde{w}, \tilde{h}_{u}, \tilde{h}_{d}, \tilde{s}\right\}$ basis, and the bottom-right block corresponds to $\tilde{s}^{\prime}$. Here $m$ broadly denotes quantities which are roughly of order the weak scale. After diagonalizing $\mathcal{M}_{\text {fermion }}$, it is clear that mixing angles of $\tilde{s}^{\prime}$ into visible sector fermions go as

$$
\theta_{\tilde{s}^{\prime} \tilde{b}} \sim \theta_{\tilde{s}^{\prime} \tilde{w}} \sim \theta_{\tilde{s}^{\prime} \tilde{h}_{u}} \sim \theta_{\tilde{s}^{\prime} \tilde{h}_{d}} \sim \theta_{\tilde{s}^{\prime} \tilde{s}} \sim \epsilon \frac{m^{\prime}}{m}
$$

up to $O(\lesssim 1)$ coefficients which are model dependent. ${ }^{3}$ These mixings induce interaction terms which involve more than one visible sector field. For example, if the visible sector superpotential contains a term $\kappa S^{3} / 3$, then this mixing leads to an interaction $-2 \theta_{\tilde{s}^{\prime} \tilde{s}} \kappa s \tilde{s} \tilde{s}^{\prime}$.

We next consider the hidden sector scalars. As in the case of fermions, we can remove the kinetic mixing between $s$ and $s^{\prime}$ at the leading order in $\epsilon$ via the shift

$$
s^{\prime} \rightarrow s^{\prime}-\epsilon s .
$$

This induces interaction terms $-\epsilon s\left(\partial / \partial s^{\prime}\right) \mathcal{L}_{\text {hid }}$, each of which contains only a single visible sector field.

Interactions involving more than one visible sector scalar predominantly arise from the $F_{S}^{\dagger} F_{S^{\prime}}$ term in eq. (3.3). By expanding both $F_{S}^{\dagger}$ and $F_{S^{\prime}}$ to first order in field fluctuations, we find

$$
\mathcal{L}_{\text {kin }} \supset \epsilon\left(\lambda v\left(h_{u} \cos \beta+h_{d} \sin \beta\right)+\frac{d^{2} f(x)}{d x^{2}} s\right)\left(m^{\prime} s^{\prime}\right)^{\dagger},
$$

which mixes $h_{u}, h_{d}$, and $s$ with $s^{\prime}$ with coefficients of order $\epsilon m m^{\prime}$. Consequently, the scalar mass-squared matrix is schematically

$$
\mathcal{M}_{\text {scalar }}^{2} \approx\left(\begin{array}{c|c}
m^{2} \approx(\text { weak scale })^{2} & \epsilon m m^{\prime} \\
\hline \epsilon m m^{\prime} & m^{\prime 2}
\end{array}\right),
$$

where the upper-left block corresponds to the neutral Higgs fields of the visible sector, and the bottom-right block corresponds to $s^{\prime}$. Here, the basis of scalars is spanned by both CP even and odd components. In the case that $\mathrm{CP}$ is conserved, $\mathcal{M}_{\text {scalar }}^{2}$ decomposes into CP even and CP odd blocks. Interestingly, we find that the mixing angles of $s^{\prime}$ with the visible sector states scale as in the fermion sector:

$$
\theta_{s^{\prime} h_{u}} \sim \theta_{s^{\prime} h_{d}} \sim \theta_{s^{\prime} s} \sim \epsilon \frac{m^{\prime}}{m}
$$

\footnotetext{
${ }^{3}$ To be precise, the $\theta$ parameters here represent the fractions of $\tilde{b}, \tilde{w}, \tilde{h}_{u}, \tilde{h}_{d}, \tilde{s}$ which contain the "mostly $\tilde{s}^{\prime}$ mass eigenstate," which is purely $\tilde{s}^{\prime}$ at the leading order in $\epsilon$.
} 
where $h_{u}$, etc., collectively denote the $\mathrm{CP}$ even and $\mathrm{CP}$ odd components. Note that $s^{\prime}$ does not mix into (the longitudinal component of) the $Z$ boson as dictated by gauge invariance, which implies that $\theta_{s^{\prime} h_{u}} / \theta_{s^{\prime} h_{d}} \propto \cot \beta$ for the CP odd component of $s^{\prime}$.

We finally comment on possible effects of supersymmetry breaking. If the scale of supersymmetry breaking in the hidden sector, $\tilde{m}^{\prime}$, is larger than the scale $m^{\prime}$ in eq. (4.1), then the mass scale of the hidden sector will be set by $\tilde{m}^{\prime}$ (at least for the scalars). Moreover, mixing angles between $s^{\prime}$ and visible sector scalars receive an extra contribution of order

$$
\delta \theta_{s^{\prime} h_{u}} \sim \delta \theta_{s^{\prime} h_{d}} \sim \delta \theta_{s^{\prime} s} \sim \epsilon\left(\frac{\tilde{m}^{\prime}}{m}\right)^{2},
$$

since the soft supersymmetry breaking mass-squared matrix obtains a nonvanishing $s-s^{\prime}$ component of order $\epsilon \tilde{m}^{\prime 2}$ after the shift of eq. (4.5). For $\tilde{m}^{\prime} \gg m^{\prime}$, this contribution may be larger than that in eq. (4.8).

To summarize, we find that the portal between the visible and hidden sectors takes the form

$$
\mathcal{L}_{\text {portal }}=\mathcal{L}_{\text {portal in }}+\mathcal{L}_{\text {portal out }}+\text { h.c. }
$$

where

$$
\begin{aligned}
\mathcal{L}_{\text {portal in }} & =\left(-\epsilon \tilde{s} \frac{\partial}{\partial \tilde{s}^{\prime}}-\epsilon s \frac{\partial}{\partial s^{\prime}}\right) \mathcal{L}_{\text {hid }}, \\
\mathcal{L}_{\text {portal out }} & =\left(\tilde{s}^{\prime} \sum_{\tilde{\phi}} \theta_{\tilde{s}^{\prime}} \frac{\partial}{\partial \tilde{\phi}}+s^{\prime} \sum_{\phi} \theta_{s^{\prime} \phi} \frac{\partial}{\partial \phi}\right) \mathcal{L}_{\text {vis }} .
\end{aligned}
$$

Here, $\mathcal{L}_{\text {hid }}$ and $\mathcal{L}_{\text {vis }}$ denote interaction Lagrangians of the hidden and visible sectors, respectively, while $\tilde{\phi}$ and $\phi$ run over the visible sector neutralinos and neutral Higgs states, respectively. ${ }^{4}$ The mixing angles $\theta_{\tilde{s}^{\prime} \tilde{\phi}}$ and $\theta_{s^{\prime} \phi}$ are given by eqs. (4.4) and (4.8), and are all of order $\epsilon \mathrm{m}^{\prime} / \mathrm{m}$ (unless the contribution of eq. (4.9) is larger). As we will see, supersymmetric cascades at colliders will mainly portal in to the hidden sector via $\mathcal{L}_{\text {portal in }}$ and portal out of the hidden sector via $\mathcal{L}_{\text {portal out }}$. Thus, these processes are controlled by interaction terms with coefficients of order $\epsilon$ and $\epsilon m^{\prime} / m$, respectively.

\section{To the hidden sector}

In this section, we discuss the collider signatures associated with the decay of the LOSP into the hidden sector via the singlet portal. In theories with $R$-parity conservation, superparticles produced at colliders will cascade down to lighter $R$-parity odd particles. Since

\footnotetext{
${ }^{4}$ The second term of eq. (4.12) is only schematic, as the mixing angles for CP even and odd components differ in general. For the CP-conserving case, the precise expression is given by$$
\mathcal{L}_{\text {portal out }} \supset\left(s_{R}^{\prime} \sum_{\phi_{R}} \theta_{s_{R}^{\prime} \phi_{R}} \frac{\partial}{\partial \phi_{R}}+s_{I}^{\prime} \sum_{\phi_{I}} \theta_{s_{I}^{\prime} \phi_{I}} \frac{\partial}{\partial \phi_{I}}\right) \mathcal{L}_{\text {vis }},
$$

where $s^{\prime}=\left(s_{R}^{\prime}+i s_{I}^{\prime}\right) / \sqrt{2}$, and $\phi_{R}$ and $\phi_{I}$ represent real and imaginary components of the visible sector Higgs fields, respectively. In the general case with CP violation, both of the sums run over $\phi_{R}$ and $\phi_{I}$, i.e. both $s_{R}^{\prime}$ and $s_{I}^{\prime}$ mix with all the visible sector Higgs fields.
} 


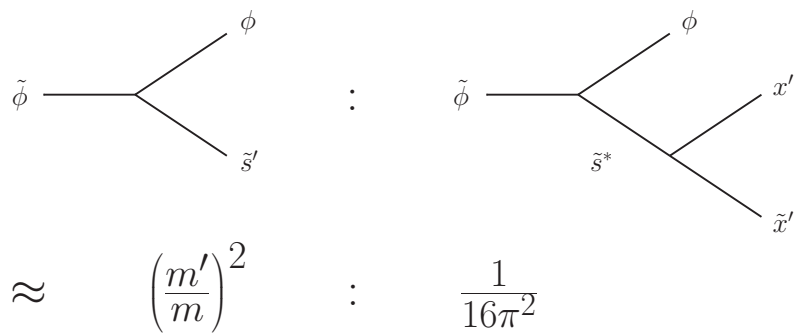

Figure 2. Decays of the Higgsino, squark, or slepton LOSP, represented collectively by $\tilde{\phi}$. Here $\phi$ denotes a Higgs (or electroweak gauge) boson, quark, or lepton, while $x^{\prime}$ and $\tilde{x}^{\prime}$ denote hidden sector fields to which $\tilde{s}^{\prime}$ couples with sizable strength.

the hidden sector typically contains an $R$-parity odd state lighter than the LOSP, these cascades produce hidden sector particles.

We first consider the case in which the LOSP is the lightest neutralino. Since the bino or wino does not couple directly to the hidden sector, the singlino and Higgsino components are most relevant.

The singlino component leads to an invisible decay $\tilde{s} \rightarrow x^{\prime} \tilde{x}^{\prime}$ through an $\epsilon$-suppressed coupling in eq. (4.11), where $x^{\prime}$ and $\tilde{x}^{\prime}$ are hidden sector particles to which $\tilde{s}^{\prime}$ couples with sizable strength. It also leads to a subdominant decay mode $\tilde{s} \rightarrow h \tilde{x}^{\prime}, s \tilde{x}^{\prime}$ through an $\epsilon m^{\prime} / m$-suppressed coupling in eq. (4.12), where $h$ represents either a neutral Higgs or $Z$ boson. In particular, this leads to the (standard model like) Higgs boson in the final state of the $\tilde{s}$ decay with a probability of $O\left(\mathrm{~m}^{\prime 2} / \mathrm{m}^{2}\right)$. Note that the singlino is produced only through the interaction of eq. (1.3), so that the Higgs boson is also produced with a probability of $O\left(\gtrsim 1 / 16 \pi^{2}\right)$ when a heavier superparticle decays into the singlino.

The Higgsino component, on the other hand, leads to either a two-body decay through $\tilde{h} \rightarrow h \tilde{s}^{\prime}, s \tilde{s}^{\prime}$ via an $\epsilon m^{\prime} / m$-suppressed coupling, or a three-body decay through $\tilde{h} \rightarrow h \tilde{s}^{*} \rightarrow$ $h x^{\prime} \tilde{x}^{\prime}$ via an off-shell $\tilde{s}$ and an $\epsilon$-suppressed coupling. These processes have competitive rates, with a ratio $\Gamma_{\tilde{h} \rightarrow h \tilde{s}^{\prime}, s \tilde{s}^{\prime}} / \Gamma_{\tilde{h} \rightarrow h x^{\prime} \tilde{x}^{\prime}} \approx 16 \pi^{2} m^{\prime 2} / m^{2}$, which depends strongly on the size of $m^{\prime}$; see figure 2. Note that decay through the Higgsino component always leads to the Higgs boson with an $O(1)$ probability, regardless of the size of $m^{\prime}$.

We next consider the case of a chargino LOSP. As before, the charged wino component does not couple directly to the hidden sector, so that only the charged Higgsino component is relevant. Similarly to the neutral component, the charged Higgsino decays as $\tilde{h}^{ \pm} \rightarrow h^{ \pm} \tilde{s}^{\prime}, h^{ \pm} x^{\prime} \tilde{x}^{\prime}$ with competitive rates, where $h^{ \pm}$represents either a charged Higgs or $W$ boson.

We now consider the case in which the LOSP is a squark, slepton, or sneutrino. In this case, the LOSP decays to a quark, lepton or neutrino, plus invisible decay products, as depicted in figure 2. If the Yukawa couplings of the LOSP are large, then the LOSP decays to an off-shell Higgsino which then mixes into a hidden sector singlino $\tilde{s}^{\prime}$, or an offshell singlino $\tilde{s}^{*}$ decaying into hidden sector states $x^{\prime} \tilde{x}^{\prime}$. Alternatively, if the LOSP Yukawa couplings are small, then the LOSP decays to an off-shell gaugino which is converted to the Higgsino and then to either $\tilde{s}^{\prime}$ or $x^{\prime} \tilde{x}^{\prime}$. Either way, the rates to $\tilde{s}^{\prime}$ and $x^{\prime} \tilde{x}^{\prime}$ are competitive, with the ratio again given by $\approx 16 \pi^{2} \mathrm{~m}^{\prime 2} / \mathrm{m}^{2}$. 
If the LOSP is the gluino or (almost) pure bino or wino, then it decays through an off-shell sfermion. The final state is then the same as the corresponding sfermion decay, with an extra quark, lepton, or neutrino.

In summary, the above analysis highlights a number of salient points. First, the visible products of a supersymmetric cascade can be different from conventional supersymmetric theories. For example, if the LOSP is the lightest neutralino in which the Higgsino fraction is larger than the singlino one, then its decay leads to the Higgs boson with an $O(1)$ fraction of the time, even if the LOSP is not Higgsino-like. This leads to a distinct signature in which an $O(1)$ fraction of the supersymmetric events is accompanied by the Higgs boson. While it is possible to mimic this in a conventional scenario, e.g. by having the Higgsinolike next-to-lightest supersymmetric particle decaying into the gravitino, observing many Higgs bosons may be an important first step in identifying the present scenario.

Second, since MSSM states interact with the hidden sector only through the Higgs sector, supersymmetric cascades are required to pick up a Higgs VEV or emit a physical (neutral or charged) Higgs boson (or the corresponding longitudinal electroweak gauge boson). Given that the existence of cascades containing a charged Higgs boson typically implies the existence of cascades containing a neutral Higgs boson, ${ }^{5}$ we should expect a Higgs boson in the visible products of supersymmetric cascades with

$$
\frac{\# \text { of SUSY events with } h}{\# \text { of SUSY events }} \approx O\left(10^{-2}-1\right)
$$

where the Higgs boson is typically produced at the end of the visible sector cascade. This is because any cascade decay process involving a Higgs VEV is necessarily accompanied by the corresponding subleading process in which the Higgs VEV is replaced by a physical on-shell Higgs boson, which is suppressed by an additional $1 / 16 \pi^{2}$ phase space factor. This implies that there is a minimum rate for high transverse-momentum Higgs production associated with significant missing energy, which may help to discover the Higgs boson through the $b \bar{b}$ decay mode [31].

Finally, note that LOSP decays will produce hidden sector scalars $\left(s^{\prime}\right.$ or $\left.x^{\prime}\right)$ in a significant fraction of events, $O\left(\gtrsim 1 / 16 \pi^{2}\right)$. Indeed, even if the LOSP has a dominant branching fraction to $\tilde{s}^{\prime}$, there is typically a competitive decay mode to $x^{\prime} \tilde{x}^{\prime}$ through an off-shell $\tilde{s}$. This fact can have a significant implications for the "portal out" of the hidden sector discussed in the next section.

\section{From the hidden sector}

As we have seen, the characteristic mass scale of the hidden sector is less than or of order the weak scale. In fact, the dynamically induced scale $\Lambda_{\text {eff }}$ in eq. (3.5) may be significantly smaller than the weak scale due to $\sin 2 \beta$ suppression. Here we assume that the hidden sector scale is indeed below the superpartner threshold. In this case, hidden sector states

\footnotetext{
${ }^{5}$ An exception to this arises in a special case in which a light top squark can decay only to the bottom and a charged Higgsino, which in turn decays into an (off-shell) charged Higgs boson. There is then no corresponding process which yields a neutral Higgs boson.
} 
will be produced in supersymmetric cascades, and may return via decays into standard model particles.

Since the hidden sector couples to the visible sector through the Higgs sector, the dominant final state is generically the heaviest standard model particles which are kinematically accessible. Whether these return processes indeed occur at colliders may depend on the spectrum of the hidden sector. For example, if cascades produce only hidden sector states which are stable, e.g. the lightest supersymmetric particle, then there will be no return process. However, as we have seen in the previous section, supersymmetric cascades typically produce hidden sector scalars with a significant fraction, which in turn decay back to the standard model. In particular, in the minimal theory defined in section $4, s^{\prime}$ scalars are directly produced via portal in. Since $s^{\prime}$ couples to the standard model through mixing with the Higgs field, it necessarily decays to standard model particles. ${ }^{6}$

Let us now consider decay of the $s^{\prime}$ scalars. For definiteness, we assume that the Higgs sector preserves $\mathrm{CP}$ and consider the CP even component (real part) of $s^{\prime}$. We assume that the mass of $s^{\prime}$, which we denote here by $m^{\prime}$ regardless of its origin, is below $100 \mathrm{GeV}$; the case $m^{\prime} \gtrsim 100 \mathrm{GeV}$ will be discussed later. The terms relevant to the decay are then

$$
\mathcal{L}_{\text {portal out }} \supset s^{\prime}\left(\theta_{s^{\prime} h_{u}} \frac{\partial}{\partial v_{u}}+\theta_{s^{\prime} h_{d}} \frac{\partial}{\partial v_{d}}\right) \mathcal{L}_{\mathrm{SM}} .
$$

Here we have defined

$$
\begin{aligned}
\mathcal{L}_{\mathrm{SM}}= & -\frac{1}{4 e^{2}\left(v_{u}, v_{d}\right)} F_{\mu \nu} F^{\mu \nu}-\frac{1}{4 g_{\mathrm{S}}^{2}\left(v_{u}, v_{d}\right)} G_{\mu \nu}^{a} G^{a \mu \nu} \\
& -v_{u}\left(\sum_{i} y_{u_{i}} \bar{u}_{i} u_{i}\right) \\
& -v_{d}\left(\sum_{i} y_{d_{i}} \bar{d}_{i} d_{i}+\sum_{i} y_{\ell_{i}} \bar{\ell}_{i} \ell_{i}\right)
\end{aligned}
$$

which is simply the standard model Lagrangian at the scale $m^{\prime}$ written as a function of the Higgs VEVs. Here $e$ and $g_{\mathrm{s}}$ are the electromagnetic and QCD couplings renormalized to the scale $m^{\prime}$. These couplings depend on $v_{u}$ and $v_{d}$ due to one-loop renormalization effects from standard model fermions which are heavier than $s^{\prime}$, since the masses of these heavy particles depend on $v_{u}$ and $v_{d}$. The sum over up-type quarks $u_{i}$, down-type quarks $d_{i}$, and charged leptons $\ell_{i}$ runs over states which are lighter than $s^{\prime}$ and thus kinematically allowed in the $s^{\prime}$ decay. As expected, $s^{\prime}$ couples to light fermions through Yukawa couplings, and to gauge bosons via loops of heavy fermions.

When $m^{\prime}$ is above the QCD scale $\Lambda_{\mathrm{QCD}}$, it is reasonable to compute decay rates to gluons and quarks at the partonic level using $\mathcal{L}_{\mathrm{SM}}$. However, for $m^{\prime} \lesssim \Lambda_{\mathrm{QCD}}, s^{\prime}$ no longer decays to constituent partons but to hadrons. To estimate the hadronic branching ratio

\footnotetext{
${ }^{6}$ If $s^{\prime}$ is heavier than $\tilde{s}^{\prime}$, then $s^{\prime}$ may decay into $\tilde{s}^{\prime}$ and the gravitino. For gravity mediation, the decay rate is very small $\Gamma_{s^{\prime} \rightarrow \tilde{s}^{\prime} \tilde{G}} \simeq m^{5} / 16 \pi F^{2}$, where $\sqrt{F}$ is the scale of fundamental supersymmetry breaking. For gauge mediation, $s^{\prime}$ and $\tilde{s}^{\prime}$ are nearly degenerate, $\delta m^{\prime} \approx \max \left\{\epsilon^{2} m^{\prime}, \epsilon^{2} m^{2} / 16 \pi^{2} m^{\prime}\right\} \ll m^{\prime}$, so that the decay rate $\Gamma_{s^{\prime} \rightarrow \tilde{s}^{\prime} \tilde{G}} \simeq m^{\prime} \delta m^{\prime 4} / \pi F^{2}$ is again suppressed. In either case, the partial decay rate to the gravitino is much smaller than the dominant one to standard model particles.
} 


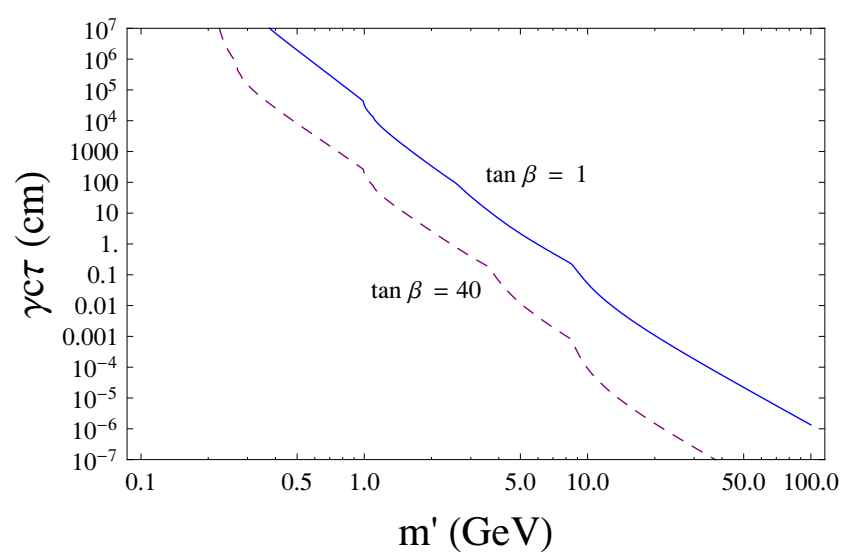

Figure 3. Decay length $\gamma c \tau$ of the hidden sector singlet $s^{\prime}$ as a function of its mass $m^{\prime}$. Here the solid (blue) and dashed (purple) lines correspond to $\tan \beta=1$ and 40, respectively. For concreteness, we have used a boost factor $\gamma=m / m^{\prime}$ and mixing angles $\theta_{s^{\prime} h_{u}}=\theta_{s^{\prime} h_{d}}=\epsilon m^{\prime} / m$ where $m=300 \mathrm{GeV}$ and $\epsilon=10^{-2}$.

in this mass range, we replace the terms involving $g / u / d / s$ in $\mathcal{L}_{\mathrm{SM}}$ with the $\mathrm{SU}(3)_{f}$ chiral Lagrangian describing the dynamics of octet mesons. We interpolate between the partonic theory and the chiral Lagrangian at the charm threshold $m^{\prime}=2 m_{c}$. See [32] for the details of this calculation.

Having obtained the couplings of $s^{\prime}$ to standard model fields, we can now compute the decay length and branching ratios of $s^{\prime}$. In figure 3, we show the decay length $c \tau$ multiplied by a boost factor $\gamma$ as a function of $m^{\prime}$. For illustrative purposes, we have taken $\gamma=m / m^{\prime}$ and $\theta_{s^{\prime} h_{u}}=\theta_{s^{\prime} h_{d}}=\epsilon m^{\prime} / m$ where $m=300 \mathrm{GeV}$ and $\epsilon=10^{-2}$. (Of course, quantities represented by $m$ in $\gamma, \theta_{s^{\prime} h_{u}}$ and $\theta_{s^{\prime} h_{d}}$ are not the same; $\gamma$ even varies event by event.) The scaling of the decay length with respect to these parameters is given by $\gamma c \tau \propto \gamma \theta_{s^{\prime} h_{u, d}}^{-2} \propto \epsilon^{-2}\left(m / m^{\prime}\right)^{3}$.

The branching ratios for $s^{\prime}$ decay as a function of $m^{\prime}$ are shown in figures 4 and 5 for $\tan \beta=1$ and 40, respectively. Here, we have taken $\theta_{s^{\prime} h_{u}} / \theta_{s^{\prime} h_{d}}=1$; all the dependencies on other free parameters cancel in the branching ratios. We can see that $s^{\prime}$ decays generically to the heaviest possible state kinematically available, although there are some exceptions, e.g. see $2 m_{\tau}<m^{\prime}<2 m_{b}$ for small $\tan \beta$. The dependence on $\tan \beta$ appears clearly in the leptonic branching ratios for $m^{\prime}>2 m_{\mu}$, which arises from the fact that the ratios of the Yukawa couplings $y_{u_{i}} / y_{\ell_{i}}$ depend on $\tan \beta$. Note that these branching ratios, however, are not uniquely fixed by $\tan \beta$; they also depend on $\theta_{s^{\prime} h_{u}} / \theta_{s^{\prime} h_{d}}$ which we have taken to be unity for illustrative purposes. The rare branching ratio into photons is also strongly affected by $\tan \beta$.

Figure 3 shows that for $m^{\prime} \lesssim O(10 \mathrm{GeV}), s^{\prime}$ is long-lived in collider timescales, leading to a displaced decay vertex from which standard model particles originate. The decay product typically consists of two particles, e.g. $e^{+} e^{-}, \mu^{+} \mu^{-}$, or $\pi^{+} \pi^{-}$, with a small opening angle of $O\left(\mathrm{~m}^{\prime} / \mathrm{m}\right)$. The direction of these particles point almost to the decay vertex, since the intermediate $s^{\prime}$ is highly boosted with $\gamma \approx O\left(\mathrm{~m} / \mathrm{m}^{\prime}\right)$. 


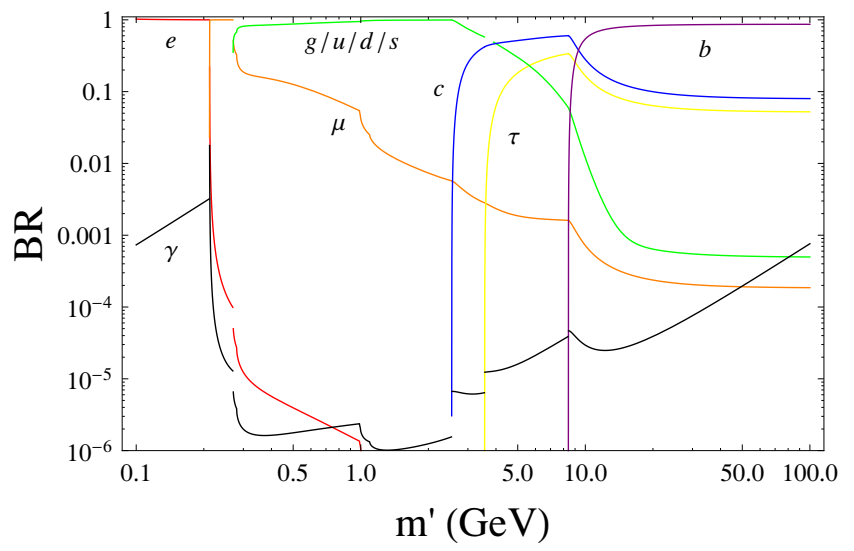

Figure 4. Branching ratios of the hidden sector singlet $s^{\prime}$ into $\{e, \mu, \tau, g / u / d / s, c, b, \gamma\}$, corresponding to the $\{$ red, orange, yellow, green, blue, purple, black $\}$ lines, as a function of $m^{\prime}$. Here, we have taken $\theta_{s^{\prime} h_{u}}=\theta_{s^{\prime} h_{d}}$ and $\tan \beta=1$. Below the $2 m_{c}$ threshold, decays to partonic $g / u / d / s$ are replaced by decays to octet mesons.

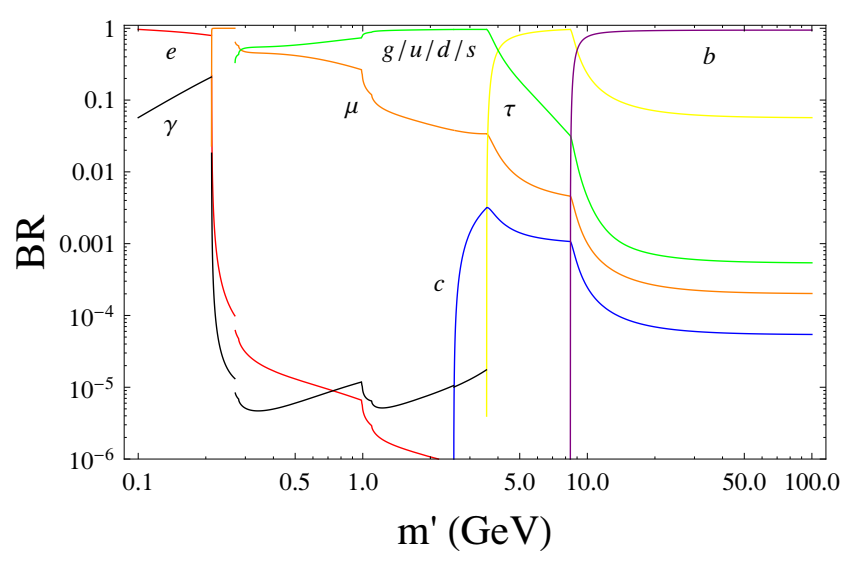

Figure 5. The same plot as in figure 4 except for $\tan \beta=40$.

The decay of $s^{\prime}$ may be measured if it occurs inside a detector. To obtain a sufficient number of events, e.g. $N_{\min } \approx$ a few, we need

$$
n \varepsilon \sigma_{\mathrm{SUSY}} \int \mathcal{L} \min \left\{1, \frac{L}{\gamma c \tau}\right\} \gtrsim N_{\min },
$$

where $n, \varepsilon, \sigma_{\text {SUSY }}$, and $\int \mathcal{L}$ are the effective number of $s^{\prime}$ per supersymmetric event, signal acceptance after cuts, total superparticle production cross section, and integrated luminosity, respectively. The last factor in the left-hand-side represents a fraction of $s^{\prime}$ decay occurring inside a detector with size $L$. Taking $n \varepsilon \approx 0.1, \sigma_{\mathrm{SUSY}} \approx O(10 \mathrm{pb})$, and $\int \mathcal{L} \approx O\left(100 \mathrm{fb}^{-1}\right)$, for example, eq. (6.3) gives $\gamma c \tau \lesssim O\left(10^{6}-10^{7} \mathrm{~cm}\right)$ for $L \approx 1 \mathrm{~m}$. We therefore expect that the $s^{\prime}$ decay can be seen at the LHC for a significant parameter region of $m^{\prime} \gtrsim O(1 \mathrm{GeV})$, assuming that the background is under control.

If most of $s^{\prime}$ decays inside a detector, i.e. $\gamma c \tau \lesssim L$, then we may get a large number of $s^{\prime}$ decay events $N_{\mathrm{dec}}=n \varepsilon \sigma_{\mathrm{SUSY}} \int \mathcal{L}$. In this case, a rare decay mode into two photons may be observable if $N_{\text {dec }} \gtrsim O\left(10^{5}-10^{6}\right)$. 
For heavier $s^{\prime}$ with $m^{\prime} \gtrsim O(10 \mathrm{GeV})$, the $s^{\prime}$ decays promptly. For $m^{\prime} \lesssim 130 \mathrm{GeV}$, many supersymmetric events will be accompanied by one or two pairs of standard model particles - mostly $b \bar{b}, c \bar{c}$, or $\tau^{+} \tau^{-}$, but also $\mu^{+} \mu^{-}$with $O\left(10^{-4}\right)$ probability. In contrast to the small $m^{\prime}$ case, opening angles for these pairs are not very small. For $m^{\prime} \gtrsim 130 \mathrm{GeV}$, $s^{\prime}$ decays (often dominantly) into a pair of electroweak gauge bosons (on- or off-shell), although it may also decay into $t \bar{t}$ or two Higgs bosons. The branching ratios into these modes depend on $\tan \beta$ and $\theta_{s^{\prime} h_{u}} / \theta_{s^{\prime} h_{d}}$.

Finally, we discuss decays of the $\mathrm{CP}$ odd component of $s^{\prime}$. The $\mathrm{CP}$ even and odd components of $s^{\prime}$ typically have comparable decay lengths and branching ratios for $\Lambda_{\mathrm{QCD}}<$ $m^{\prime} \lesssim 130 \mathrm{GeV}$ (except that the $\mathrm{CP}$ odd mixing angle $\theta_{s^{\prime} h_{u}}$ has a $\cot \beta$ suppression at large $\tan \beta$ ). For $m^{\prime} \gtrsim 130 \mathrm{GeV}$, however, the $\mathrm{CP}$ odd component decays dominantly to either $b \bar{b}$, $t \bar{t}$, or the Higgs and $Z$ bosons, instead of two electroweak or Higgs bosons. For $m^{\prime} \lesssim \Lambda_{\mathrm{QCD}}$, the leading hadronic decay of the CP odd component is to three rather than two mesons, and is thus suppressed by three-body phase space.

If $\mathrm{CP}$ is violated in the Higgs sector, the mass eigenstates are not the same as $\mathrm{CP}$ eigenstates. In this case, both $s^{\prime}$ mass eigenstates decay generically through the faster of the CP even and odd decay modes.

\section{Constraints}

The hidden sector singlets and singlinos are additional neutral scalars and fermions which feebly interact with visible sector fields. As such, they are constrained, albeit weakly, by existing experimental bounds from light axion, Higgs, and neutralino searches.

Our discussion will largely hinge on the magnitude of the mixing angles $\theta_{s^{\prime} h_{u, d}}$ and $\theta_{\tilde{s}^{\prime} \tilde{h}_{u, d}}$, as defined in eqs. (4.4) and (4.8). Parametrically, these mixing angles scale as

$$
\begin{aligned}
\theta_{s^{\prime} h_{u, d}} & \sim \theta_{\tilde{s}^{\prime} \tilde{h}_{u, d}} \sim \epsilon \frac{m^{\prime}}{m} \\
& \simeq 3 \times 10^{-5}\left(\frac{\epsilon}{10^{-2}}\right)\left(\frac{m^{\prime}}{1 \mathrm{GeV}}\right)\left(\frac{300 \mathrm{GeV}}{m}\right) .
\end{aligned}
$$

Since they are naturally small, theories with singlet portal are constrained only weakly.

For $m^{\prime}$ smaller than a few $\mathrm{GeV}$, dominant constraints come from axion and light scalar searches. In most cases, appropriate constraints can be estimated by replacing the axion decay constant $f_{a}$ in the axion bounds (for $\tan \beta \approx 1$ ) as

$$
\frac{1}{f_{a}} \rightarrow \max \left\{\frac{\theta_{s^{\prime} h_{u}}}{v_{u}}, \frac{\theta_{s^{\prime} h_{d}}}{v_{d}}\right\}
$$

For $m^{\prime} \lesssim 30 \mathrm{MeV}$, bounds from globular cluster stars, white dwarfs, and SN 1987A require $f_{a} \gtrsim 10^{9} \mathrm{GeV}[33,34]$. We then find from eq. (7.2) that $\epsilon$ needs to be somewhat small, e.g. $\epsilon \lesssim 10^{-3}$, for $m^{\prime} \approx O(10 \mathrm{MeV}) .^{7}$ The complimentary regions in $m^{\prime}$, either $\ll 10 \mathrm{MeV}$ or $\gtrsim 100 \mathrm{MeV}$, is not constrained by astrophysics.

\footnotetext{
${ }^{7}$ Another possibility to evade the bounds for $m^{\prime} \approx O(10 \mathrm{MeV})$ is to have large $\epsilon \gtrsim 1 / \tan \beta$, leading to $f_{a} \lesssim 10^{6} \mathrm{GeV}$. In this case, $s^{\prime}$ produced in SN 1987A is trapped inside the supernova, so that it does not carry away significant energy. Constraints from other astrophysical observations are irrelevant for these values of $m^{\prime}$.
} 
For $m^{\prime}<m_{K}-m_{\pi} \simeq 350 \mathrm{MeV}$, rare processes such as $K^{ \pm} \rightarrow \pi^{ \pm} s^{\prime}$ may give a constraint. Since $s^{\prime}$ in this mass range is long-lived, the corresponding axion bound is $f_{a} \gtrsim 100 \mathrm{TeV}$ [35]. Given eqs. (7.1) and (7.2), however, this constraint is easily evaded.

For $2 m_{\mu}<m^{\prime}<m_{B}-m_{K} \simeq 4.8 \mathrm{GeV}$, the leading constraints come from rare decays of $B$ mesons, $B \rightarrow K s^{\prime} \rightarrow K \mu^{+} \mu^{-}$. These decays place a stringent bound on the corresponding effective axion decay constant $f_{a} \gtrsim$ few $\times 10^{3} \mathrm{TeV}[36,37]$. Since the processes occur mainly with $s^{\prime}$ emitted from internal top quarks, this translates into $\theta_{s^{\prime} h_{u}} \lesssim 10^{-4}$, obtained with $1 / f_{a}$ replaced by $\theta_{s^{\prime} h_{u}} / v_{u}$, rather than eq. (7.2). In view of eq. (7.1), this bound is satisfied in most of natural parameter regions.

If the hidden singlet is heavier, $m^{\prime}>m_{B}-m_{K}$, then constraints may still arise from LEP results, e.g. from bounds on $s^{\prime}$-strahlung and gauge boson fusion into $s^{\prime}$. However, the cross sections for these $s^{\prime}$ production processes relative to the corresponding neutral Higgs boson production go roughly as $\theta_{s^{\prime} h_{u, d}}^{2}$, so that they are typically very small. Constraints from anomalous $Z$ boson decays are also easily satisfied, since $Z \rightarrow h^{*} s^{\prime} \rightarrow b \bar{b} s^{\prime}$ goes as $\theta_{s^{\prime} h_{u, d}}^{2}$ while $Z \rightarrow s^{\prime} s^{\prime}$ and $\tilde{s}^{\prime} \tilde{s}^{\prime}$ as $\theta_{s^{\prime} h_{u, d}}^{4}$ and $\theta_{\tilde{s}^{\prime} \tilde{h}_{u, d}}^{4}$, respectively.

\section{Dark matter}

So far we have focused our attention on a simple hidden sector consisting of a single superfield $S^{\prime}$. In general, however, the hidden sector may have a much richer structure. In particular, if it contains a particle which is stable on cosmological timescales, then that particle may comprise all of or a component of dark matter. In this section, we discuss possible implications of such hidden sector dark matter.

As a simple example, let us consider the theory defined in eq. (3.6), augmented by $\mathbb{Z}_{2}$ stabilized dark matter, $H^{\prime}$ :

$$
W_{\text {hid }}=-\Lambda_{\text {eff }}^{2} S^{\prime}+\frac{\kappa^{\prime}}{3} S^{\prime 3}+\frac{\lambda^{\prime}}{2} S^{\prime} H^{\prime 2}
$$

This superpotential has a supersymmetry-preserving minimum at $\left\langle S^{\prime}\right\rangle=\sqrt{\Lambda_{\text {eff }}^{2} / \kappa^{\prime}}$ and $\left\langle H^{\prime}\right\rangle=0$. After supersymmetry breaking is communicated to the hidden sector, $\left\langle S^{\prime}\right\rangle$ will shift but $\left\langle H^{\prime}\right\rangle$ can still be vanishing. The lightest component of $H^{\prime}$ is then stable dark matter, whose mass, $m_{\mathrm{DM}}$, is given by the larger of $\lambda^{\prime}\left\langle S^{\prime}\right\rangle \approx O\left(\Lambda_{\text {eff }}\right)$ and gravity mediated contributions.

Which component of $\left\langle H^{\prime}\right\rangle$ is the lightest depends on details of supersymmetry breaking. In high-scale mediation scenarios, e.g. gravity mediation, both scalar and fermion components may receive sizable masses from supersymmetry breaking, which can be as large as the weak scale. In gauge mediation, all components of $H^{\prime}$ are nearly degenerate, as supersymmetry is approximately preserved in the hidden sector. Small mass splittings, however, can be generated at order $\epsilon^{2}$. The largest effect typically comes from $\left\langle F_{S^{\prime}}\right\rangle \approx O\left(\epsilon^{2} m^{3} / 16 \pi^{2} m^{\prime}\right)$ induced by a supersymmetry breaking tadpole for $s^{\prime}$, in which case dark matter is a real scalar field which is lighter than the other components by $O\left(\left\langle F_{S^{\prime}}\right\rangle / m_{\mathrm{DM}}\right)$. While these mass splittings are small, typically of $O(100 \mathrm{keV}-10 \mathrm{GeV})$ for $\epsilon=10^{-2}$, heavier components may still decay into lighter before today, depending on parameters (emitting either 
a gravitino or a pair of standard model particles). If the decay into the gravitino is not kinematically allowed, then both the fermion and lighter scalar components are absolutely stable (due to $\mathbb{Z}_{2} \times R$-parity).

The precise relic abundance of dark matter depends on the hidden sector spectrum, including mass splittings among components of $H^{\prime}$. For $\kappa^{\prime} \lesssim \lambda^{\prime}$, it is roughly controlled by the thermally-averaged annihilation cross section into fields in the $S^{\prime}$ multiplet $\langle\sigma v\rangle \approx$ $\lambda^{\prime 4} / 64 \pi m_{\mathrm{DM}}^{2}$, giving

$$
\Omega_{\mathrm{th}} \approx 0.1\left(\frac{0.1}{\lambda^{\prime}}\right)^{4}\left(\frac{m_{\mathrm{DM}}}{10 \mathrm{GeV}}\right)^{2}
$$

Thus a stable particle(s) residing in the $H^{\prime}$ multiplet can comprise all or a significant fraction of the dark matter in the universe. ${ }^{8}$

The scattering cross section of $H^{\prime}$ dark matter with a target nucleus is dominated by $t$-channel exchange of CP even $s^{\prime}[38]$, and is given by

$$
\sigma_{T}=\frac{\mu_{T}^{2}}{2 \pi} \frac{\lambda^{\prime 2}}{m^{\prime 4}}\left(Z g_{s^{\prime} p p}+(A-Z) g_{s^{\prime} n n}\right)^{2}
$$

for both fermion and scalar components, $h^{\prime}$ and $\tilde{h}^{\prime}$. Here, $Z$ and $A$ are the atomic number and weight of the target nucleus, $\mu_{T}$ is the dark matter-nucleus reduced mass, and $g_{s^{\prime} N N}$ for $N=p, n$ is the coupling of $s^{\prime}$ to the proton and neutron:

$$
g_{s^{\prime} N N}=\frac{m_{N}}{\sqrt{2} v}\left(\frac{\theta_{s^{\prime} h_{u}}}{\sin \beta} \sum_{q=u, c, t} f_{q}^{N}+\frac{\theta_{s^{\prime} h_{d}}}{\cos \beta} \sum_{q=d, s, b} f_{q}^{N}\right) .
$$

Using the following nucleon parameters [39]

$$
\begin{array}{llll}
f_{u}^{p}=0.023, & f_{d}^{p}=0.034, & f_{s}^{p}=0.14, & f_{c, b, t}^{p}=0.059, \\
f_{u}^{n}=0.019, & f_{d}^{n}=0.041, & f_{s}^{n}=0.14, & f_{c, b, t}^{n}=0.059,
\end{array}
$$

we obtain the spin-independent dark-matter scattering cross section per nucleon, defined by $\left.\sigma_{T}\right|_{A=Z=1}{ }^{9}$

$$
\begin{aligned}
\sigma \simeq 2 \times 10^{-48} & \mathrm{~cm}^{2} \frac{(1+1.6 \tan \beta)^{2}}{\sin ^{2} \beta}\left(\frac{\epsilon}{10^{-2}}\right)^{2} \\
& \times\left(\frac{\lambda^{\prime}}{0.1}\right)^{2}\left(\frac{10 \mathrm{GeV}}{m^{\prime}}\right)^{2}\left(\frac{300 \mathrm{GeV}}{m}\right)^{2} .
\end{aligned}
$$

Here, we have taken $\theta_{s^{\prime} h_{u}}=\theta_{s^{\prime} h_{d}}=\epsilon m^{\prime} / m$ for illustrative purposes. The cross section of eq. (8.6) is typically beyond the reach of current experiments, but it can be significantly enhanced at large $\tan \beta$ (and large $\lambda^{\prime}$ and $\theta_{s^{\prime} h_{d}}$ ).

\footnotetext{
${ }^{8}$ We assume that $\tilde{s}^{\prime}$ annihilates into $s^{\prime}$ with a sufficiently large cross section or decays into $s^{\prime}$ and the gravitino so that it does not overclose the universe. Indeed, this condition is satisfied in most of natural parameter regions.

${ }^{9}$ An alternative definition for the dark matter-nucleon cross section is sometimes used, see e.g. [40], but the difference is negligible for $g_{s^{\prime} p p} \approx g_{s^{\prime} n n}$ and the dark matter sufficiently heavier than the nucleon.
} 


\section{Discussion and conclusions}

If the standard model is merely one of many sectors embedded in some fundamental theory - as is often the case in string theory - then these additional sectors may be "hidden" in the sense that at low energies they interact only weakly with standard model particles. In theories with weak scale supersymmetry, the characteristic mass scales associated with these hidden sectors can be naturally at or below the weak scale, generated through supersymmetry breaking effects. Moreover, supersymmetry may offer a unique window into these hidden sectors via the decay of the LOSP, which can provide a rich phenomenology at colliders.

In this paper, we have considered a scenario in which the visible and hidden sectors both contain singlet chiral superfields, $S$ and $S^{\prime}$, which, through a marginal kinetic mixing operator, connect the otherwise sequestered sectors. This operator spontaneously induces a light mass scale $\Lambda_{\text {eff }} \approx O(0.1-100 \mathrm{GeV})$ in the hidden sector. Supersymmetric cascades necessarily produce Higgs bosons in an $O(0.01-1)$ fraction of events, and typically exhibit displaced decays of a light hidden sector state into standard model particles.

The theories discussed here may be easily discriminated from theories in which the lightest hidden sector particle is a hidden U(1) gauge boson kinetically mixed with the photon. If the mass of the hidden sector singlet is greater than the muon threshold, $m^{\prime}>2 m_{\mu}$, then the singlet portal gives the branching ratio to electrons versus muons $\operatorname{Br}\left(s^{\prime} \rightarrow e^{+} e^{-}\right) / \operatorname{Br}\left(s^{\prime} \rightarrow \mu^{+} \mu^{-}\right) \approx\left(m_{e} / m_{\mu}\right)^{2} \ll 1$, while the hidden gauge boson case leads to a comparable branching ratio [26]. For $m^{\prime}<2 m_{\mu}$, the singlet portal yields a sizable decay rate to photons $\operatorname{Br}\left(s^{\prime} \rightarrow \gamma \gamma\right) \gtrsim 10^{-3}$, while the rate to (three) photons is negligibly small in the gauge kinetic mixing scenario.

On the other hand, it may not be trivial to distinguish the singlet portal from a generic theory in which the lightest hidden sector particle is a scalar, $\phi^{\prime}$. In such a theory, the two-body decay of the lightest hidden sector particle typically yields heavy standard model fermions, $\operatorname{Br}\left(\phi^{\prime} \rightarrow f \bar{f}\right) \propto m_{f}^{2}$, since this process requires a helicity flip in a final state fermion. However, in the case that the visible and hidden sectors are connected via a $\mathrm{U}(1)$ gauge kinetic mixing - the only alternative to singlet kinetic mixing involving a marginal portal interaction - the two-body decay of $\phi^{\prime}$ (via a loop of the hidden gauge boson, $\gamma^{\prime}$, and $f$ ) is always accompanied by a four-body decay (via a tree diagram with two off-shell $\left.\gamma^{\prime}\right)$. The branching ratio of these two processes scales as $\operatorname{Br}\left(\phi^{\prime} \rightarrow f \bar{f}\right) / \operatorname{Br}\left(\phi^{\prime} \rightarrow\right.$ $\left.\gamma^{\prime *} \gamma^{\prime *} \rightarrow f \bar{f} f \bar{f}\right) \approx m_{f}^{2} m_{\gamma^{\prime}}^{4} / m_{\phi^{\prime}}^{6}$, so that the four-body decay rate may be significant, giving a different set of signatures for the portal out. In more general cases, a detailed analysis of supersymmetric cascades may be required - for example, to discriminate from other theories, e.g. the ones considered in [41]. In those cases, other characteristic features of the singlet portal, e.g. Higgs bosons arising from the end of the visible sector part of the cascades, will be important for identifying the underlying theory.

The analysis of the present paper can be straightforwardly extended to the case of multiple hidden sectors containing singlet chiral superfields, all kinetically mixed. In this setup, visible sector superparticles typically cascade into the hidden sector which has the largest kinetic mixing with the visible sector. The produced states may then cascade decay 
into states in another hidden sector or perhaps back to the visible sector. These cascades will in general terminate at stable final states which are either hidden sector or standard model particles. Signatures of multiple hidden sector theories are, therefore, similar to the ones discussed in this paper.

Finally, let us present another class of singlet portal theories in which the hidden sector singlet is odd under $R$-parity. We consider a hidden sector singlet $N^{\prime}$ that kinetically mixes with right-handed neutrinos, $N$ :

$$
\mathcal{L}=\epsilon \int d^{4} \theta N^{\dagger} N^{\prime}+\text { h.c. },
$$

where we have omitted the generation index of $N$. Here the fermionic components of $N \supset n$ and $N^{\prime} \supset n^{\prime}$ are the right-handed neutrino and the hidden sector singlino, respectively. The right-handed neutrinos will have standard Majorana masses as well as neutrino Yukawa couplings

$$
W=\frac{M}{2} N^{2}+y_{\nu} L H_{u} N
$$

leading to small neutrino masses, $m_{\nu}=y_{\nu}^{2} v_{u}^{2} / M$, through the seesaw mechanism. (Of course, Dirac neutrinos, $M=0$ and $y_{\nu} \ll 1$, are also an option.)

In this scenario, the kinetic mixing terms in eq. (9.1) do not induce an effective Polonyi term for the hidden sector, since the sleptons do not acquire VEVs. Consequently, spontaneous generation of scales does not occur in theories of $R$-parity odd singlet kinetic mixing.

On the other hand, the neutrino portal in eq. (9.1) can lead to distinctive signatures at colliders. As in the case of $R$-parity even singlet kinetic mixing, supersymmetric cascades which originate in the visible sector invariably traverse into the hidden sector. This typically yields a Higgs, lepton, or neutrino at the bottom of the visible sector part of the cascades. Meanwhile, any hidden sector singlino $n^{\prime}$ produced in the process will decay back via $n^{\prime} \rightarrow \ell^{ \pm} h^{\mp}, \nu h$ with a macroscopic displacement, where $h^{ \pm}$and $h$ represent on or off-shell Higgs or electroweak gauge bosons. Whether these return processes indeed occur is model dependent, as in the case of other kinetic mixing portals. For example, if $W_{\text {hid }} \supset N^{\prime} H^{\prime 2}$ or $N^{\prime 3}$, then the portal back may occur. (The latter breaks $R$-parity.) Here the mass scale of the hidden sector can be generated by gravity mediated contributions, which are of order or perhaps smaller than the weak scale.

The decay of $n^{\prime}$ is mediated by operators induced via mass mixing between $n$ and $n^{\prime}$, analogous to the $\tilde{s} / \tilde{s}^{\prime}$ singlino case. The mixing angles are

$$
\theta_{n^{\prime} n} \sim \epsilon \frac{m^{\prime}}{M}
$$

where $m^{\prime}$ is the mass of $n^{\prime}$. This gives the decay width for $n^{\prime} \rightarrow \ell^{ \pm} W^{\mp}$

$$
\Gamma_{n^{\prime} \rightarrow \ell^{ \pm} W \mp} \sim \frac{1}{8 \pi} \frac{\epsilon^{2} y_{\nu}^{2} m^{\prime 3}}{M^{2}}=\frac{1}{8 \pi} \frac{\epsilon^{2} m_{\nu} m^{\prime 3}}{v_{u}^{2} M},
$$

assuming that the final state $W$ boson is on-shell. (If not, the width is suppressed further.) The decay length for the return process is then

$$
\gamma c \tau \sim 10^{7} \mathrm{~cm}\left(\frac{10^{-2}}{\epsilon}\right)^{2}\left(\frac{200 \mathrm{GeV}}{m^{\prime}}\right)^{3}\left(\frac{M}{10^{8} \mathrm{GeV}}\right),
$$


where we have used $m_{\nu}=0.1 \mathrm{eV}$. This provides the possibility of observing the portal out process at the LHC for $M$ as large as $\approx O\left(10^{8} \mathrm{GeV}\right)$ — close to the scale suggested by thermal leptogenesis [42]. Unfortunately, the portal in process is often a slow, three-body decay, reducing the reach of $M$ by about two orders of magnitude, but the maximal reach can be obtained, e.g., if a sneutrino is the LOSP. Note that the decay of $n^{\prime}$ is not helicity suppressed, so the final state lepton can provide direct information on the flavor structure for $\epsilon, y_{\nu}$, and $M$.

More generally, visible return processes arising from supersymmetric hidden sector cascades can provide a unique and powerful probe of visible sector physics at very high energies. The case of $R$-parity odd singlet kinetic mixing is a particular instance of employing highly displaced vertices to extend the reach of the LHC to extremely high energies. Another example allowing for such a probe is given by a hidden sector $\mathrm{U}(1)$ gauge field which kinetically mixes with a heavy $\mathrm{U}(1)_{B-L}$ or $\mathrm{U}(1)_{\text {flavor }}$ gauge boson, but not with $\mathrm{U}(1)$ hypercharge (although the reach is generically lower than the neutrino case). In fact, this method can also apply to $R$-parity even singlet kinetic mixing, with the singlet $S$ having a large supersymmetric mass.

\section{Acknowledgments}

This work was supported in part by the Director, Office of Science, Office of High Energy and Nuclear Physics, of the US Department of Energy under Contract DE-AC0205CH11231, and in part by the National Science Foundation under grants PHY-0555661 and PHY-0855653.

Open Access. This article is distributed under the terms of the Creative Commons Attribution Noncommercial License which permits any noncommercial use, distribution, and reproduction in any medium, provided the original author(s) and source are credited.

\section{References}

[1] S.B. Giddings, S. Kachru and J. Polchinski, Hierarchies from fluxes in string compactifications, Phys. Rev. D 66 (2002) 106006 [hep-th/0105097] [SPIRES].

[2] S. Dimopoulos, S. Kachru, N. Kaloper, A.E. Lawrence and E. Silverstein, Small numbers from tunneling between brane throats, Phys. Rev. D 64 (2001) 121702 [hep-th/0104239] [SPIRES].

[3] A. Arvanitaki, S. Dimopoulos, S. Dubovsky, N. Kaloper and J. March-Russell, String axiverse, Phys. Rev. D 81 (2010) 123530 [arXiv:0905.4720] [SPIRES].

[4] A. Arvanitaki, N. Craig, S. Dimopoulos, S. Dubovsky and J. March-Russell, String photini at the LHC, Phys. Rev. D 81 (2010) 075018 [arXiv: 0909.5440] [SPIRES].

[5] C. Cheung, Y. Nomura and J. Thaler, Goldstini, JHEP 03 (2010) 073 [arXiv:1002.1967] [SPIRES].

[6] N. Craig, J. March-Russell and M. McCullough, The goldstini variations, JHEP 10 (2010) 095 [arXiv: 1007.1239] [SPIRES]. 
[7] B. Holdom, Two U(1)'s and epsilon charge shifts, Phys. Lett. B 166 (1986) 196 [SPIRES].

[8] S.L. Glashow, Positronium versus the mirror universe, Phys. Lett. B 167 (1986) 35 [SPIRES].

[9] E.D. Carlson and S.L. Glashow, Nucleosynthesis versus the mirror universe, Phys. Lett. B 193 (1987) 168 [SPIRES].

[10] B. Holdom, Oblique electroweak corrections and an extra gauge boson, Phys. Lett. B 259 (1991) 329 [SPIRES].

[11] R. Foot and X.-G. He, Comment on Z Z-prime mixing in extended gauge theories, Phys. Lett. B 267 (1991) 509 [SPIRES].

[12] K.R. Dienes, C.F. Kolda and J. March-Russell, Kinetic mixing and the supersymmetric gauge hierarchy, Nucl. Phys. B 492 (1997) 104 [hep-ph/9610479] [SPIRES].

[13] M. Pospelov, A. Ritz and M.B. Voloshin, Secluded WIMP dark matter, Phys. Lett. B 662 (2008) 53 [arXiv:0711.4866] [SPIRES].

[14] N. Arkani-Hamed, D.P. Finkbeiner, T.R. Slatyer and N. Weiner, A theory of dark matter, Phys. Rev. D 79 (2009) 015014 [arXiv:0810.0713] [SPIRES].

[15] M. Pospelov, Secluded U(1) below the weak scale, Phys. Rev. D 80 (2009) 095002 [arXiv:0811.1030] [SPIRES].

[16] E.J. Chun and J.-C. Park, Dark matter and sub-GeV hidden U(1) in GMSB models, JCAP 02 (2009) 026 [arXiv: 0812.0308] [SPIRES].

[17] Y. Bai and Z. Han, Measuring the dark force at the LHC, Phys. Rev. Lett. 103 (2009) 051801 [arXiv:0902.0006] [SPIRES].

[18] C. Cheung, J.T. Ruderman, L.-T. Wang and I. Yavin, Kinetic mixing as the origin of light dark scales, Phys. Rev. D 80 (2009) 035008 [arXiv:0902.3246] [SPIRES].

[19] A. Katz and R. Sundrum, Breaking the dark force, JHEP 06 (2009) 003 [arXiv:0902.3271] [SPIRES].

[20] B. Batell, M. Pospelov and A. Ritz, Probing a secluded U(1) at B-factories, Phys. Rev. D 79 (2009) 115008 [arXiv:0903.0363] [SPIRES].

[21] R. Essig, P. Schuster and N. Toro, Probing dark forces and light hidden sectors at low-energy $e^{+} e^{-}$colliders, Phys. Rev. D 80 (2009) 015003 [arXiv:0903.3941] [SPIRES].

[22] M. Reece and L.-T. Wang, Searching for the light dark gauge boson in GeV-scale experiments, JHEP 07 (2009) 051 [arXiv:0904.1743] [SPIRES].

[23] D.E. Morrissey, D. Poland and K.M. Zurek, Abelian hidden sectors at a GeV, JHEP 07 (2009) 050 [arXiv:0904.2567] [SPIRES].

[24] J.D. Bjorken, R. Essig, P. Schuster and N. Toro, New fixed-target experiments to search for dark gauge forces, Phys. Rev. D 80 (2009) 075018 [arXiv:0906.0580] [SPIRES].

[25] B. Batell, M. Pospelov and A. Ritz, Exploring portals to a hidden sector through fixed targets, Phys. Rev. D 80 (2009) 095024 [arXiv:0906.5614] [SPIRES].

[26] N. Arkani-Hamed and N. Weiner, LHC signals for a superunified theory of dark matter, JHEP 12 (2008) 104 [arXiv:0810.0714] [SPIRES].

[27] M. Baumgart, C. Cheung, J.T. Ruderman, L.-T. Wang and I. Yavin, Non-abelian dark sectors and their collider signatures, JHEP 04 (2009) 014 [arXiv:0901.0283] [SPIRES]. 
[28] J. Polchinski and L. Susskind, Breaking of supersymmetry at intermediate-energy, Phys. Rev. D 26 (1982) 3661 [SPIRES].

[29] U. Ellwanger, Nonrenormalizable interactions from supergravity, quantum corrections and effective low-energy theories, Phys. Lett. B 133 (1983) 187 [SPIRES].

[30] J. Bagger, E. Poppitz and L. Randall, Destabilizing divergences in supergravity theories at two loops, Nucl. Phys. B 455 (1995) 59 [hep-ph/9505244] [SPIRES].

[31] G.D. Kribs, A. Martin, T.S. Roy and M. Spannowsky, Discovering the Higgs boson in new physics events using jet substructure, Phys. Rev. D 81 (2010) 111501 [arXiv:0912.4731] [SPIRES].

[32] R.S. Chivukula, A.G. Cohen, H. Georgi and A.V. Manohar, Couplings of a light Higgs boson, Phys. Lett. B 222 (1989) 258 [SPIRES].

[33] G.G. Raffelt, Astrophysical methods to constrain axions and other novel particle phenomena, Phys. Rept. 198 (1990) 1 [SPIRES].

[34] Particl Data Group collaboration, K. Nakamura et al., Review of particle physics, J. Phys. G 37 (2010) 075021.

[35] J. Mardon, Y. Nomura and J. Thaler, Cosmic signals from the hidden sector, Phys. Rev. D 80 (2009) 035013 [arXiv:0905.3749] [SPIRES].

[36] B. Batell, M. Pospelov and A. Ritz, Multi-lepton signatures of a hidden sector in rare B decays, arXiv:0911.4938 [SPIRES].

[37] M. Freytsis, Z. Ligeti and J. Thaler, Constraining the axion portal with $B \rightarrow K \ell^{+} \ell^{-}$, Phys. Rev. D 81 (2010) 034001 [arXiv:0911.5355] [SPIRES].

[38] D.P. Finkbeiner, T.R. Slatyer and N. Weiner, Nuclear scattering of dark matter coupled to a new light scalar, Phys. Rev. D 78 (2008) 116006 [arXiv:0810.0722] [SPIRES].

[39] M. Drees and M. Nojiri, Neutralino-nucleon scattering revisited, Phys. Rev. D 48 (1993) 3483 [hep-ph/9307208] [SPIRES].

[40] J.D. Lewin and P.F. Smith, Review of mathematics, numerical factors and corrections for dark matter experiments based on elastic nuclear recoil, Astropart. Phys. 6 (1996) 87 [SPIRES].

[41] M.J. Strassler, Possible effects of a hidden valley on supersymmetric phenomenology, hep-ph/0607160 [SPIRES].

[42] W. Buchmüller, R.D. Peccei and T. Yanagida, Leptogenesis as the origin of matter, Ann. Rev. Nucl. Part. Sci. 55 (2005) 311 [hep-ph/0502169] [SPIRES]. 